\title{
Achieving Greater Real-world Impact of Research Outputs: It's not Rocket Science
}

\author{
$\underline{\text { K. Lowell }}^{\text {a }}$ \\ ${ }^{a}$ CRC for Spatial Information/University of Melbourne, Carlton, Victoria, AUSTRALIA \\ Email: klowell@crcsi.com.au
}

\begin{abstract}
Real-world uptake of research results is partially dependent on internal cultures of three types of research institutions. Their prevailing research cultures are characterised herein by their scientific mission, funding, targeted end-user, and time required to produce research results. The characterisations are generalisations that are nonetheless broadly applicable and useful for the issues raised in this paper. The table below summarises relevant aspects of the research cultures of the entities considered.
\end{abstract}

\begin{tabular}{|c|c|c|c|c|}
\hline $\begin{array}{l}\text { Research } \\
\text { Entity }\end{array}$ & Mission & Funding & Targeted end-user & $\begin{array}{c}\text { Time to Produce } \\
\text { Results }\end{array}$ \\
\hline Universities & $\begin{array}{l}\text { Generate } \\
\text { fundamental } \\
\text { knowledge }\end{array}$ & $\begin{array}{l}\text { Competitive } \\
\text { grants }\end{array}$ & $\begin{array}{lr}\text { Other } & \text { scientists } \\
\text { with } & \text { real-world } \\
\text { users } & \text { being } \\
\text { "eventual" } & \text { end- } \\
\text { users } & \end{array}$ & $\begin{array}{l}\text { Longer time to produce } \\
\text { outputs than other } \\
\text { entities because } \\
\text { research outputs are not } \\
\text { time-sensitive }\end{array}$ \\
\hline $\begin{array}{c}\text { Public- } \\
\text { Government } \\
\text { Institutions }\end{array}$ & $\begin{array}{l}\text { Serve the needs of } \\
\text { government }\end{array}$ & $\begin{array}{l}\text { Core internal } \\
\text { funding } \\
\text { supplemented by } \\
\text { partnerships }\end{array}$ & $\begin{array}{l}\text { Government and } \\
\text { partners }\end{array}$ & $\begin{array}{l}\text { Rapid for time-sensitive } \\
\text { questions; relatively } \\
\text { rapid for partner-based } \\
\text { research. }\end{array}$ \\
\hline $\begin{array}{l}\text { Collaborativ } \\
\text { e Research } \\
\text { Entities } \\
\text { (CREs) }\end{array}$ & $\begin{array}{l}\text { Facilitate } \\
\text { collaborative research } \\
\text { among universities, } \\
\text { government agencies, } \\
\text { and private industry }\end{array}$ & $\begin{array}{l}\text { Core funding } \\
\text { from government } \\
\text { supplemented by } \\
\text { member } \\
\text { contributions }\end{array}$ & $\begin{array}{l}\text { CRE members who } \\
\text { are real-world end- } \\
\text { users themselves } \\
\text { and connected to } \\
\text { others }\end{array}$ & $\begin{array}{l}\text { Variable depending on } \\
\text { partners and nature of } \\
\text { individual research } \\
\text { activities }\end{array}$ \\
\hline
\end{tabular}

It is argued that the implicit assumption that better science - and the associated advancement of sciencedriven knowledge from a state of "complete ignorance" to "perfect knowledge" -- will necessarily lead to more adoption is incorrect. In fact, it is suggested that the greatest opportunity for adoption of research outputs occurs when one first moves from a state of "complete ignorance" because the marginal gains in realworld outcomes are much greater at this point than when knowledge advances from a state of being, for example, "90\% complete." Moreover, it is suggested that as knowledge about a system/phenomenon advances, there is an "upper limit of practical utility" (ULPU) beyond which additional knowledge does not enhance opportunities for end-user adoption. This is because of both the potential economic costs of adopting more advanced science outputs, and the reality that in many situations once scientific knowledge has reached a certain level, considerations other than research results become at least as important in decision-making. Moreover, the marginal cost of advancing knowledge increases considerably above the ULPU making the cost-benefit ratio of additional research increasingly unattractive if the research goal is real-world adoption.

These factors are embodied in the title of this article which is a tongue-in-cheek reaction to the implicit belief that improved science - i.e., "rocket science" - is the key to better adoption. While better science outputs may be a necessary condition for a certain level of adoption, they are not a sufficient condition at all levels i.e., above the ULPU. Hence the key to better adoption is not rocket science alone, but a combination of factors including some that are affected by different research cultures.

This article is based on the author's $25+$ years of experience in a variety of research institutions.

Keywords: $\quad$ Research adoption, institutional research cultures, data-based science, model embodiment 


\section{INTRODUCTION}

Scientific research is considered vital for the general well-being of society. Science is considered critical for maintaining the production of essential goods and services, distributing them efficiently, and assessing tradeoffs among competing needs and allocations of resources.

This is as true for landscape and natural resource management as it is for other sectors such as health care, energy, and manufacturing. That landscapes are expected to produce a broad range of "commodities" for society's benefit is reflected in the relatively recent coinage of the term "ecosystem services." That is, in addition to providing the ecosystem service of economic production associated with activities such as agriculture and forestry, landscapes are expected to produce biodiversity, clean water, and others.

Given this state of affairs, one would expect the linkage between research outputs and real-world landscape management to be strong and seamless. After all, landscape management continues to be the subject of considerable amounts of research. And such research has an implicit or explicit goal of influencing onground activity for individual farms and larger areas such as catchments.

While some landscape management research does eventually achieve on-ground impact directly or indirectly, the transfer of research outputs to end-users and the use of science-based knowledge could be improved. This is evidenced by, among others, this special MODSIM session that explores effective adoption. That is, if adoption were currently considered optimal, there would be no need for such a session.

This paper examines a number of aspects of real-world research adoption based on the author's $25+$ years of international experience as a university-based academic, a principle scientist in a state government organization, and a program director in a federally funded cooperative research centre. It considers motivations for science and its adoption from institutional and individual perspectives. It concludes by examining the general nature of scientific inquiry and how it impacts the adoptability of research outputs.

\section{SCIENCE-BASED INSTITUTIONS AND THEIR SCIENTISTS}

In this section, the research cultures of universities, public/government institutions, and collaborative research entities are discussed. Each is discussed in generalities with the acknowledgement that the generalisations made are not universally applicable across an organisation's entire research culture, nor across different organisations of the same type. However, the generalisations are useful characterisations of different research cultures to examine effective adoption of research outputs for landscape management.

Research cultures in each type of organisation are characterised by three factors that strongly impact adoption of research results: research funding, the targeted end-user, and the time required to produce results.

\subsection{Universities}

Universities are considered here only for the role as research institutions - i.e., their educational role is not addressed.

Universities are seen as repositories of knowledge. This has led to a prevailing research mission of the generation of fundamental knowledge - i.e., "knowledge for the sake of knowledge." University research culture understandably reflects and is structured around this core mission.

Research funding: Universities are most focussed on obtaining funding from competitive grants programs such as those administered by national science agencies like the Australian Research Council (ARC), the Natural Science Engineering and Research Council (NSERC) in Canada, and the National Science Foundation (NSF) in the United States. Funds are won from such agencies largely on the strength of a research idea and the research productivity of the project leader - the latter of which is primarily determined by the number and of refereed publications and the quality of outlets in which they appear.

Targeted end-user: The pursuit of fundamental knowledge defines the immediate end-user as other scientists who collectively comprise the research/knowledge community. Research results reach this user-group through refereed publications that also serve as a constantly expanding and updated record of the current state of knowledge. The imperative to document contributions to the general body of scientific knowledge is coupled with the need to publish in refereed journals to win competitive grants, as well as for individual career advancement within the university. The result is that university scientists place greatest value on refereed publications in prestigious journals in part because this is the way that one demonstrates that one is reaching the immediate end-user targeted: other scientists. 
University scientists are also aware - and hopeful - that in the long-term the impact of their research results may extend beyond the science community. That is, there is an awareness that the immediate end-user other scientists - is potentially not the eventual or ultimate end-user. However, in university research culture, serving the ultimate end-user is secondary to generating fundamental knowledge.

Time to produce results: The production of fundamental knowledge is usually not time-sensitive. Hence university research culture emphasises scientific rigour over timely output. Coupled with this is the need to train younger researchers - a factor that decreases the speed by which research results are produced.

It is acknowledged that university research culture is more complex than this simplistic synopsis although most academic scientists associated with landscape management would identify with the elements discussed. Important exceptions are those domains and researchers focussed on applied rather than fundamental research. This is addressed in due course. For now it is simply acknowledged that applied researchers are present within universities who potentially differ primarily in research funding and targeted end-user.

\subsection{Public/Government Institutions (P/GIs)}

This section refers to P/GIs associated with landscape management only. Moreover, though such organisations are multi-faceted, only their research function is considered.

The broad institutional mission of P/GIs is facilitating the establishment and execution of government policy. At times this entails being able to address a diversity of questions such as:

- What is likely to happen if...?

- How much $x$ exists currently in the landscape and how much has this changed since $y$ ?

- What is the best management option here to maximise the production of ecosystem service $x$ ?

P/GI research attempts to answer these questions to enable more informed decisions by publicly accountable institutions. Thus P/GI scientists do not generate knowledge for the sake of knowledge. They are nonetheless cognizant of the need for rigourous science and to contribute to the general body of fundamental knowledge.

Research funding: Having a mission of meeting government information needs, P/GIs receive their core funding from the government they serve. They also seek external funds two ways.

One is by providing research to organisations that would like the answer to specific questions, but that do not have an internal research infrastructure. These include private companies, agricultural cooperatives, and private foundations. Such research can be characterised as being fee-for-service or contractual, although it is generally characterised as being collaborative. In such research, the P/GIs are research providers.

A second way is in partnership with more fundamental research entities such as universities. In such cases, a key role of the P/GI is serving as a liaison between university researchers and non-scientist end-users - i.e., the ultimate end-user that is generally not directly targeted by university scientists. That being said, it is often those university researchers who are most interested in real-world outcomes who collaborate with P/GIs in this manner. In such research, P/GIs are not only research providers, but they also play an important role to connect ultimate end-users with scientists who are fundamentally interested in knowledge generation for reasons discussed in the previous section.

Targeted end-user: The end-users for P/GI research outputs are government or those that the government wants to impact, and funding partners (and their constituents) for whom research was undertaken. That is, funding partners that are industry-based may be a collective of individuals who have an expectation of a fairly direct return on investment. Research may be commissioned by such collectives on an expectation that results can be easily adopted by the individuals comprising the collective. Similarly, a government invests in P/GI research on an expectation that results will have direct value to government, or that the results will improve the ability of land managers to produce a desired ecosystem service.

Time to produce results: The institutional mission of serving government's needs means that research results must be produced more quickly by P/GIs than in a university research culture. A similar imperative exists in research commissioned by third parties such as industry groups. The ability to achieve this is dependent on the question(s) being posed. For example, regardless of the research entitiy, research results cannot be produced rapidly if a large data collection effort over multiple years is required. Conversely, critical time imperatives can lead to alternative research approaches that rely more heavily on archival information or 
modelling. Such approaches may be able to satisfy government or industry information needs while accepting the trade-off of not having purpose-collected data.

This synopsis suggests a more direct path to adoption for P/GIs than universities. However, there are two relevant considerations. First, results produced to meet government information needs may be "adopted" only by serving as input into a limited number of decisions. That is, they are not necessarily adopted by many individuals, nor are they necessarily the most important factor in a particular decision. Second, adoption of results produced specifically for landscape managers are dependent on the delivery model (as is the case for all research entities). Though much P/GIs research targets on-ground landscape managers as end-users, different P/GIs have different models for delivery, and the efficiency of delivery is dependent on many factors. For example, whereas some P/GIs may have extension officers, others may not. Similarly, some ultimate end-user groups may be well-structured and have in-built outreach programs and personnel and others may not.

\subsection{Collaborative Research Entities (CREs)}

CREs are research organisations created to link institutions and individuals from the academic, government, and private sectors. They do not exist in the research infrastructures of all nations. Where they do exist, there are generally three important characteristics. First, each is created to address a broad application sector rather than individual research question. Second, they are semi-virtual entities. That is, they have core personnel that provide administrative and overarching scientific management, but their scientists are employed by other organisations such as universities and P/GIs. Third, and most important for this article, they have an implicit or explicit goal of decreasing the time required for scientific innovation to be adopted by the ultimate real world end-users.

An important aspect of the CRE model is that it provides for and necessitates linkages among research providers and research consumers. A research provider - generally a university or P/GI - may conceive a science idea that is modified, or whose delivery is improved, based on CRE member research consumers. Alternatively, a research consumer may identify a problem whose resolution requires a structured scientific approach; working with CRE research provider members enables this to be developed and executed. In either case, the involvement of research providers and consumers in the planning and execution of a research activity is meant to improve adoption.

Research funding: Core CRE funding is provided on a competitive basis from government-based science bodies such as the Australian Research Council. Such funding, however, is considered "seed" or "partial" funding. CREs rely heavily on additional resources - both cash and in-kind - contributed by the CRE members. And indeed the willingness of potential CRE members to provide such funding is an important determinant in the competitiveness of a CRE application. Once a CRE is established, it is the personnel inkind contributions - the amount, expertise, and active involvement - that potentially has the biggest impact on adoption.

Targeted end-user: CRE members are the target end-users of CRE research outputs with the expectation that the outputs will in turn be adopted by an even wider range of users. These are most likely to be industry and government (P/GIs) members; the former are almost exclusively research consumers whereas the latter are both research producers and consumers as explained in the previous section. Universities are generally not targeted end-users, except that one of the measures used to assess the quality of CRE science is the number of refereed publications - i.e., the immediate end-user of university research outputs.

Time to produce results: Whereas industry and P/GI research consumers desire results relatively quickly, research producers - universities in particular - must address institutional missions that includes research training and contributing information to an evolving broader body of fundamental knowledge. The research training alone necessitates projects of at least three year's duration to accord with institutional requirements for doctoral degrees.

Consequently, CREs generally support a research environment that addresses the potentially conflicting timeconstraints of its member research providers and consumers. Multiple internal funding streams are developed to enable CRE members to respond to short-term opportunities, undertake activity that may be more development than research, and to pursue research whose timing is most aligned with needs of university research providers to train post-graduate students.

The CRE model would seem to be an ideal way to improve adoption of research results. And indeed that is one of the main motivations for the creation of CREs by governments. The success of this model, however, 
depends on numerous factors that are most manifested within individual CRE projects. Among these are the intangible genuine commitment of each project partner, the ability of research consumers to define their needs, and the capacity and willingness of research providers to satisfy the needs of research consumers particularly those who are not the usual users targeted by a research entity's core mission. An additional subtlety is that the way research outputs are delivered by research producers, and the ability of the research consumers to use the outputs in the way they are delivered is critical to successful adoption regardless of an overarching CRE model focussed on adoption.

\section{SCIENCE ADVANCEMENT AND ITS RELATION TO ADOPTION}

The previous section examined a variety of research cultures as a way of providing institutional context for research adoption. Implicit in these institutions and their scientists is an assumption that "better research" outputs will lead to more adoption with "better" usually being defined as research that produces outputs that have greater certainty. Examples in the context of landscape management are greater certainty about the most profitable management regime for farms in a particular area, or the future impact of climate change on biodiversity.

Underlying this is a (usually sub-conscious) belief of scientists that if one has "absolute certainty" or "perfect knowledge" then universal adoption will necessarily follow. This is the idea to which the title of this article refers. Scientists implicitly view "perfect knowledge" that can only be obtained via the "best science" - i.e., "rocket science" - as the most important way to increase adoption. It is argued, however, that science improvement is not universally the key or even necessarily important for increased adoption. Hence the solution to increased adoption of research outputs is not, in fact, "rocket science." (And the more philosophical question of whether "perfect knowledge" is even actually obtainable is not addressed.)

Figure 1 is presented as a model of how science advances with a view to adoption. It is particularly relevant for a MODSIM conference as it dichotomises science into data-based science (DBS) and modelling embodiment (ME). In the context of landscape management, DBS can be thought of simplistically as the collection of ground-based data to test a specific research hypothesis. ME is (simplistically) the compilation and intertwining of results of multiple DBS “experiments" - i.e., different DBS research results are embodied in a single "model." Though DBS and ME are often viewed as mutually exclusive or even competing science approaches, it is suggested that they are (or at least should be) mutually dependent and that they present different opportunities for adoption.

Though Figure 1 is domain-independent, it is useful to discuss it in more tangible terms. Therefore the situational scientific question is "How does Landscape Factor $Y$ respond if Factors $A, B$, and $C$ are changed?" And this translates readily into the natural language adoption question of "How should $A, B$, and/or $C$ be changed to maximise $Y$ ?"

Starting from a point of "complete ignorance" (Fig. 1, left) about the relationships among $Y$ and $A, B$, and $C$, DBS is used to establish this basic fundamental knowledge. Once a sufficient amount of DBS has been completed, ME provides a means of "filling in the gaps." That is, if $A$ was examined at three levels $A_{l}, A_{2}$, and $\left.A_{3}\right)$ at two locations of $B\left(B_{1}\right.$ and $\left.B_{2}\right)$, then modelling can provide an estimate for the impact on $Y$ of level $A_{1.5}$ at location $B_{1.5}$. This first model produced by ME can also then provide feedback about where data are sparse - i.e., where knowledge gaps are. The next round of DBS should address these gaps, which will in turn improve ME, which will in turn continue to inform DBS until, in theory, "perfect knowledge" (Fig. 1 top left) is attained. In reality, of course, DBS and ME are undertaken in parallel, and it is debatable whether or not "perfect knowledge" can actually be attained. Nonetheless, the principle of one benefiting from and informing the other is fundamental to science advancement.

The two science approaches have different benefits relative to adoption (Table 1). DBS provides for greater accuracy/precision around explicitly studied questions. However, ME provides a cost-effective way of answering questions that have not been explicitly studied. The alternative to employing ME once a certain level of knowledge is attained is to establish evermore DBS data collection activities to address all possible levels of some factor - an approach that is prohibitively expensive and excessively lengthy. Examining interactions using a DBS approach is even more resource-exhaustive particularly as interactions become more complex. ME can alternatively use literature review to compile sufficient information to describe interactions among different factors. Doing so is not dependent on having DBS for the interactions among specific conditions - geographic, climatic, species, etc. - of interest. However, there is a danger that model formulation will not take account of some unexpected interaction that has not been discovered and studied. 


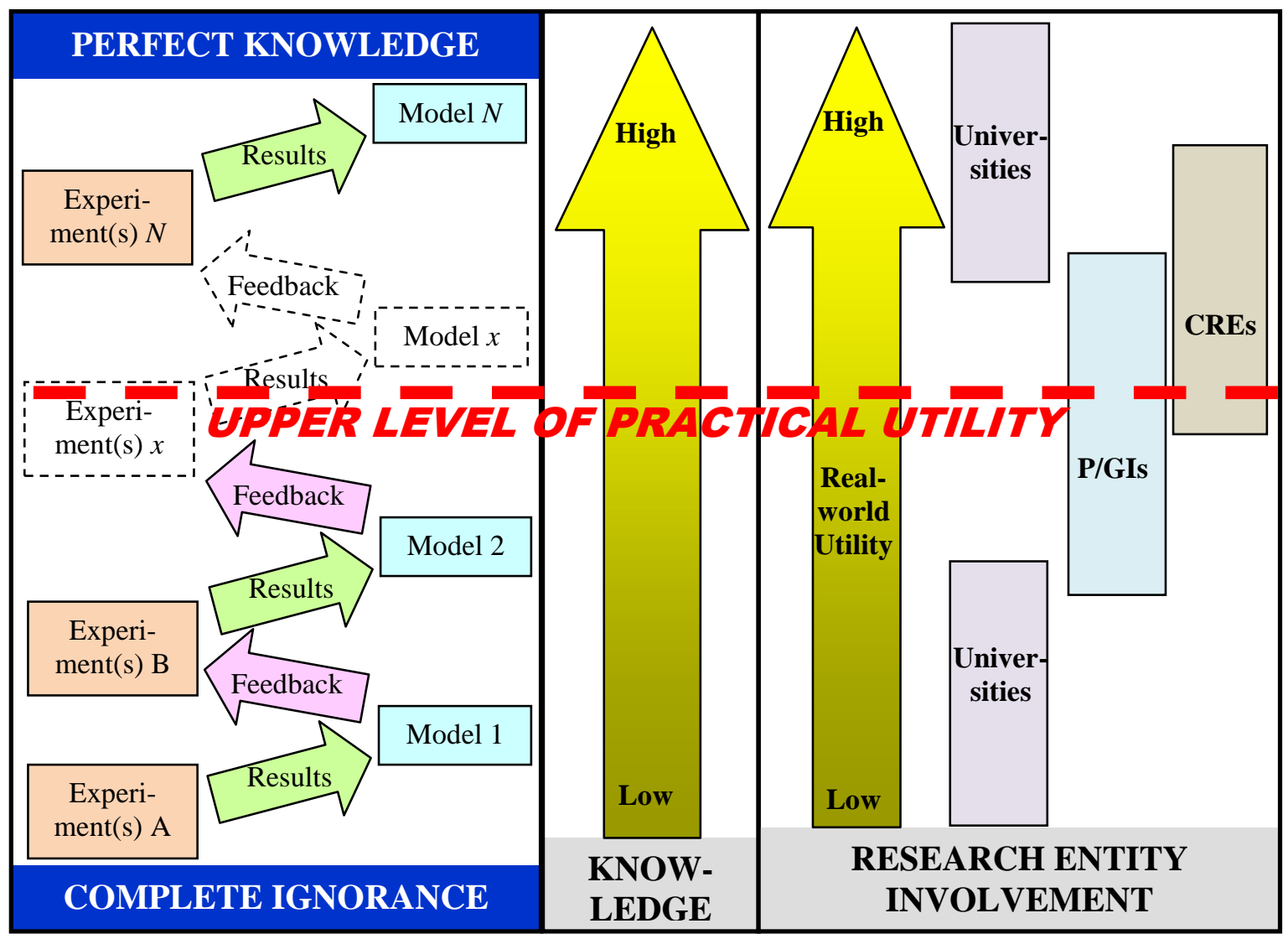

Figure 1. Science and knowledge advancement and adoption.

Table 1. Benefits of data-based science (DBS) and model embodiment (ME).

\begin{tabular}{|l|l|l|l|}
\hline \multicolumn{1}{|c|}{ Factor } & DBS & ME & \multicolumn{1}{c|}{ Comment } \\
\hline $\begin{array}{l}\text { Accuracy/precision of results for } \\
\text { explicitly studied questions }\end{array}$ & High & Low & $\begin{array}{l}\text { DBS provides greatest accuracy for the few situations } \\
\text { studied; ME “extrapolates" to a wider range but is } \\
\text { subject to a form of factor- or area-wide averaging. }\end{array}$ \\
\hline $\begin{array}{l}\text { Cost of answering questions that } \\
\text { have not been explicitly studied }\end{array}$ & High & Low & $\begin{array}{l}\text { DBS must collect more data; ME can generalise but } \\
\text { will not detect anomalous results. }\end{array}$ \\
\hline $\begin{array}{l}\text { Ability to address complex } \\
\text { interactions cost-effectively }\end{array}$ & Low & High & $\begin{array}{l}\text { ME has a risk of unknowingly modelling interactions } \\
\text { incorrectly. }\end{array}$ \\
\hline
\end{tabular}

As science advances through the DBS-ME interaction, knowledge increases and the "quality" of science outputs improves. Within one discipline, the quality of science outputs can be measured by, for example, statistical improvement -- e.g., increased $\mathrm{R}^{2}$, decreased root-mean-square-errors (RMSEs) - or the rarer discovery of something completely unexpected. (And science also advances, of course, through increased knowledge across multiple disciplines; for simplicity this is not considered here.) This improvement is accompanied by an increase in the need for experiment specificity in DBS and model complexity in ME. This highlights the mutual dependency between DBS and ME and the increased need to study interactions among all factors if one is to advance towards a state of "perfect knowledge." That is, one must examine not only the relationship of $Y$ with each of $A, B$, and $C$, but also $A$ with $B, A$ with $B$ and $C$, etc.

It is the yellow arrow in the "research entity involvement" (Fig. 1 right) that summarises the ideas presented in this article. As science progresses from a state of "complete ignorance" to "perfect knowledge," science 
outputs have more real-world utility. That is, being able to predict weather with perfect accuracy for any time period, for example, would revolutionise landscape management.

However, it is argued here that, paradoxically, real-world utility associated with "better science" is not the major determinant of adoption. Ironically the greatest opportunity for adoption is probably at the lowest knowledge levels. After all, the marginal benefits of moving from a position of "complete ignorance" to one of some "meagre" knowledge are probably much greater than moving from " $85 \%$ perfect knowledge" to "90\% perfect knowledge."

Related to this is that at some point, factors other than science outputs become more important in decisionmaking due to the need to balance real-world decisions across multiple factors. So, for example, even if "perfect knowledge science" demonstrates that all marginal agricultural land should be converted to trees to take advantage of an emerging carbon market, historical landowner values and management practices, or government policies associated with agriculture supports and viability of rural communities may preclude this from occurring.

And indeed, the data requirements associated with using "perfect knowledge science" may preclude adoption of its outputs. The primary barrier is likely to be economical - i.e., "How much will it cost to operationally implement science outputs?" As science advances the cost of collecting data needed as input to obtain "perfect knowledge" may increase, there may be a substantial initial capital outlay associated with adopting "perfect knowledge science," or the return on investment may simply not be justified. This is represented in Figure 1 by the "upper limit of practical utility" (ULPU) line. Adoptability of research results increases only to the point at which non-science considerations are most important and/or the costs associated with using better science increase prohibitively.

So where do each of the institutions examined fit into science advancement and adoption (rightmost portion of Figure 1)? University research culture is least interested in adoption. Consequently, academic scientists are likely to be involved at the most basic science level - discovery of new knowledge -- and most advanced science level - perfection of knowledge. Research activities of P/GIs will be centred on the ULPU line reflecting their use of science-based information as but one of many considerations in the establishment and implementation of public policy. Their activities are likely to overlap somewhat with university research activities. And for certain critical issues - e.g., public safety, protection of endangered faunal species - P/GIs will want "perfect knowledge." Research activities of CREs extend below the ULPU line somewhat, but are concentrated above the line. This reflects their desire to increase utilisation of "ready-for-consumer" research outputs, and also to contribute to knowledge advancement albeit within the context of producing real-world outcomes.

\section{SUMMARY}

Internal cultures of different types of research institutions impact the real-world adoption of research outputs. This is related to the overall mission of each type of research institution which in turn impacts the type of research done, the targeted end-user, and the amount of time it takes for research results to be produced. The university research mission leads to the least direct adoption path as the primary target for research outputs is other scientists with real-world users being termed "ultimate end-users" herein. P/GIs and CREs are more focussed on producing directly applicable research results, but their ability to do this is similarly impact by organisational mission and internal culture.

The fundamentals of how science advances also impacts adoption. A distinction is made between science that is driven by data collected to answer a single relatively narrow question, and science that integrates results across a number of data-driven research activities. Both strive to move knowledge of a particular phenomenon from "complete ignorance" to "perfect knowledge." Although they are often seen as competing ways of accomplishing this, they can and should function synergistically.

Underlying science advancement is often an implicit assumption that the key to adoption is "perfect knowledge" that is achieved by "perfect science" which in this paper is semi-facetiously termed "rocket science." It is argued, however, that science outputs can most be readily adopted for real-world purposes before they reach the "upper limit of practical utility" which in turn is not necessarily close to a state of "perfect knowledge." This level is related to the quality of the research outputs as well as other factors such as economics, historical practices, and government policies.

Thus once a certain base level of scientific knowledge has been attained, the key to adoption is not more or better science - i.e., the solution is not "rocket science." 